\title{
Different learning curves between stent retrieval and a direct aspiration first-pass technique for acute ischemic stroke
}

\author{
Hidehisa Nishi, MD, ${ }^{1,2}$ Akira Ishii, MD, PhD, ${ }^{1,3}$ Ichiro Nakahara, MD, PhD, ${ }^{4}$ \\ Shoji Matsumoto, MD, PhD, ${ }^{5}$ Nobutake Sadamasa, MD, PhD, ${ }^{1}$ Yasutoshi Kai, MD, PhD, ${ }^{1}$ \\ Ryota Ishibashi, MD, ${ }^{1,6}$ Michio Yamamoto, PhD, ${ }^{7}$ Satoshi Morita, PhD, ${ }^{7}$ and Izumi Nagata, MD, PhD' \\ Departments of ${ }^{1}$ Neurosurgery and ${ }^{5}$ Neurology, Kokura Memorial Hospital, Fukuoka; Departments of ${ }^{2}$ Neurosurgery and \\ ${ }^{7}$ Biomedical Statistics and Bioinformatics, Kyoto University Graduate School of Medicine, Kyoto; ${ }^{3}$ Department of Neurosurgery, \\ Kyoto University Hospital, Kyoto; ${ }^{4}$ Department of Comprehensive Strokology, Fujita Health University Medical School, Aichi; and \\ ${ }^{6}$ Department of Neurosurgery, Kurashiki Central Hospital, Okayama, Japan
}

OBJECTIVE The clinical outcomes of a direct aspiration first-pass technique (ADAPT) and stent retriever (SR) have been reported to be similar in several observational studies. In this study, procedural and clinical outcomes with ADAPT and SR for the treatment of acute ischemic stroke with large artery occlusion were compared in different time periods.

METHODS In each specific time period, SR and ADAPT were used as the first-line treatment approach for acute ischemic stroke patients with large artery occlusion at the authors' institution. Baseline characteristics, procedural variables, and functional outcome at 90 days were compared between patients treated with SR and those treated with ADAPT. These 2 groups were divided into 3 sequential subgroups to assess the learning curve effects of the endovascular team and individual operators on the procedural variables of each treatment strategy.

RESULTS Overall, 89 patients were treated. In the SR group, the recanalization rate was higher ( $84 \%$ vs $65 \% ; p=0.01$ ) and the procedure time was shorter than in the ADAPT group (median 42 minutes vs 76 minutes, $p=0.04$ ). On the subgroup analysis of the learning curve, the SR group showed more rapid improvement in procedure time than the ADAPT group ( $p=0.01$ for the team; $p<0.01$ for individual operators).

CONCLUSIONS In this initial experience, a higher recanalization rate and shorter procedure time were achieved with SR than with ADAPT. A high recanalization rate with SR was possible with relatively less clinical experience, whereas procedure time dramatically decreased with experience. These observed effects on the learning curve might be useful when choosing the method for initial endovascular treatment of acute ischemic stroke at relatively small stroke centers. https://thejns.org/doi/abs/10.3171/2017.6.JNS17872

KEY WORDS acute ischemic stroke; stent retriever; ADAPT; learning curve; vascular disorders

$\mathrm{T}$ HE efficacy of mechanical thrombectomy for treating acute ischemic stroke with large artery occlusion was demonstrated by 5 multicenter randomized clinical trials of endovascular treatment, namely MR CLEAN (Multicenter Randomized Clinical Trial of Endovascular Treatment for Acute Ischemic Stroke in the Neth- erlands), ${ }^{2}$ ESCAPE (Endovascular Treatment for Small Core and Proximal Occlusion Ischemic Stroke), ${ }^{8}$ SWIFT PRIME (Solitaire With the Intention for Thrombectomy as PRIMary Treatment for Acute Ischemic Stroke), ${ }^{14}$ EXTEND-IA (Extending the Time for Thrombolysis in Emergency Neurological Deficits with Intra-Arterial

ABBREVIATIONS ADAPT = a direct aspiration first-pass technique; ICA = internal carotid artery; IQR = interquartile range; $m R S=$ modified Rankin Scale; $m$ TICI = modified Thrombolysis in Cerebral Infarction; NIHSS = National Institutes of Health Stroke Scale; SICH = symptomatic intracerebral hemorrhage; $\mathrm{SR}=$ stent retriever . SUBMITTED April 20, 2017. ACCEPTED June 28, 2017.

INCLUDE WHEN CITING Published online January 5, 2018; DOI: 10.3171/2017.6.JNS17872. 
Therapy), ${ }^{5}$ and REVASCAT (Randomized Trial of Endovascular Revascularization With Solitaire Device Versus Best Medical Therapy in Anterior Circulation Stroke Within 8 Hours). ${ }^{9}$ Mainly, stent retrievers (SRs) were used in these trials; thus, the 2015 American Heart Association/ American Stroke Association focused update of the 2013 guidelines for the early management of patients with acute ischemic stroke recommended using SRs as mechanical thrombectomy devices. ${ }^{13}$

Conversely, Turk et al. reported a new technique that utilizes a large-bore and flexible aspiration catheter (usually the 5 MAX or 5MAX ACE catheter; Penumbra). They defined a direct aspiration first-pass technique (ADAPT) as thrombus removal using engagement with the aspiration catheter and gently pulling with continuous aspiration. ${ }^{17} \mathrm{In}$ their case series, the clinical outcomes were not inferior to those of the multicenter randomized clinical trials on using SR. In another study, Lapergue et al. compared clinical outcomes between ADAPT and the standard SR technique. They reported that ADAPT was associated with a higher recanalization rate than SR and that the procedure times and clinical outcomes were not different.10

Importantly, these clinical results with ADAPT were reported from high-volume stroke centers, and the authors did not comment on the clinical experience required to achieve those excellent clinical outcomes, which is an important factor, especially in relatively small stroke centers.

In the present study, we aimed to compare the initial clinical experience and the learning curves between the standard SR technique and ADAPT at Kokura Memorial Hospital.

\section{Methods \\ Patient Population and Study Design}

At our institution, ADAPT and SR have been used as treatment strategies since December 2013 and March 2014, respectively. All patients with acute ischemic stroke who were treated with mechanical thrombectomy using either SR or ADAPT at our institution between December 2013 and February 2016 were retrospectively reviewed. After September 2014, the stroke team determined the specific approach for first-line treatment in specific time periods: ADAPT was used as the first-line treatment strategy between September 2014 and March 2015, and the standard SR technique was used as the first-line treatment between April 2015 and January 2016. Baseline characteristics, procedural variables, periprocedural complications, and clinical outcomes were collected. The patient cohort was divided into 2 groups: 1) patients who underwent mechanical thrombectomy using SR as the initial treatment strategy (SR group); and 2) patients who underwent mechanical thrombectomy using ADAPT as the initial treatment strategy (ADAPT group).

The study protocol was approved by the institutional ethics committee of Kokura Memorial Hospital. Informed consent was not obtained from patients because this study was a retrospective analysis.

\section{Inclusion and Exclusion Criteria}

Patients with acute ischemic stroke due to occlusion of major intracranial arteries, such as the intracranial internal carotid artery (ICA), middle cerebral artery $\left(\mathrm{M}_{1}\right.$ or $\left.\mathrm{M}_{2}\right)$, basilar artery, or posterior cerebral artery $\left(\mathrm{P}_{1}\right.$ or $\mathrm{P}_{2}$ ), were treated with mechanical thrombectomy. Patients with a stroke onset within 4.5 hours received intravenous thrombolysis if there were no contraindications. Patients with tandem occlusion of the cervical carotid artery and ICA, $\mathbf{M}_{1}$, or $\mathrm{M}_{2}$ were also included in this study.

Patients with anterior circulation stroke and ASPECTS (Alberta Stroke Program Early CT Score) less than 5 and those with hemorrhagic infarction were excluded. Patients with mild symptoms defined as National Institutes of Health Stroke Scale (NIHSS) score less than 4 were also excluded. Patients for whom the time from symptom onset was more than 8 hours were excluded, except for those with wake-up stroke.

\section{End Points and Definitions}

Good clinical outcome was defined as modified Rankin Scale (mRS) scores of 0-2 at the 90-day followup. Procedure time was defined as the time from groin puncture to successful recanalization or procedure completion. Successful recanalization was defined as a modified Thrombolysis in Cerebral Infarction (mTICI) score of $2 \mathrm{~b}\left(50 \%-99 \%\right.$ reperfusion) or 3 (complete reperfusion). ${ }^{23}$ Symptomatic intracerebral hemorrhage (SICH) was defined as a type 2 parenchymal hematoma within 36 hours after treatment associated with an increase in the NIHSS score of at least 4 points from baseline (SITS-MOST [Safe Implementation of Thrombolysis in Stroke-Monitoring Study] criteria)..$^{21}$

\section{Treatment Strategy \\ Endovascular Procedure}

In all patients, a neuroendovascular team at our institution (which consisted of 9 operators) performed endovascular therapy using biplane angiography (Allura Clarity, Philips); the same endovascular team treated patients in both the SR and ADAPT groups. Procedures were performed under local anesthesia. Intravenous propofol bolus followed by drip infusion was administered to patients with agitation. Heparin was administered to maintain an activated clotting time between 200 and 300 seconds throughout the procedure. The femoral artery was catheterized with a 9-Fr sheath. If neither femoral artery could be used, the brachial artery was catheterized. For anterior circulation, a 9-Fr Optimo balloon guiding catheter (Tokai Medical Products) was the most commonly used catheter, which was placed in the cervical segment of the ICA. For posterior circulation, a 6-Fr guiding catheter was placed in the vertebral artery. The catheter was continuously flushed with heparinized saline.

\section{Stent Retrievers}

The target vessel was navigated with a microcatheter (Rebar 27 or Marksman; Medtronic) distal to the clot over a 0.014-inch microguidewire (Traxcess, MicroVention, or Chikai 14; Asahi Intecc). The Solitaire FR retrievable stent (Medtronic) or Trevo ProVue retriever (Stryker) was deployed across the site of occlusion. The stent size was se- 
lected based on the target vessel size. After device deployment, angiography was performed to evaluate immediate flow restoration. During stent retrieval, the balloon guide catheter was inflated, and continuous manual aspiration from the guiding catheter lumen was performed with a $20-\mathrm{ml}$ syringe. An intermittent catheter was not used as the first-line treatment in any patient.

\section{A Direct Aspiration First-Pass Technique}

An aspiration catheter with the largest caliber was selected based on the vessel diameter and tortuosity in each patient. Usually, a Penumbra 5MAX/ACE reperfusion catheter over a Penumbra 3MAX reperfusion catheter and a 0.014-inch microwire (Traxcess or Chikai) was used. If the Penumbra 5MAX/ACE was considered too large, a Penumbra 4MAX reperfusion catheter over a Neurodeo/ PX SLIM (Penumbra) and 0.014-inch microwire was used. The aspiration catheter was positioned adjacent to the clot and was connected to the Penumbra aspiration pump for 2 minutes. If the blood could not be retrieved through the catheter, the catheter was slowly withdrawn while maintaining aspiration.

\section{Assessment of the Learning Curve Effect on the Treatment Strategies}

The learning curve effect according to the team's experience and the individual operator's experiences were analyzed. Each group was divided into 3 sequential subgroups, and procedural variables (i.e., recanalization rate, procedure time, $\mathrm{SICH}$ ) between subgroups were statistically analyzed to estimate the learning curve effects of SR and ADAPT on procedural variables.

\section{Statistical Methods}

Variables are presented as the mean $\pm \mathrm{SD}$, count and percentage, or median with interquartile range (IQR). Categorical variables were compared using the chi-square test and likelihood ratio test, and the odds ratio was calculated for each variable. Continuous variables were compared using the Student t-test or Wilcoxon signed-rank test. Shifts in the distributions of the mRS scores at 90 days were also analyzed using a proportional odds model (shift analysis) that contained terms for age, site of the occluded vessels, presence of tandem occlusion, arterial fibrillation, and use of tissue plasminogen activator.

To compare outcomes in the entire study population, adjusted estimates of the effects for each end point were also calculated using multiple logistic regression with adjustment for age, site of the occluded vessels, presence of tandem occlusion, arterial fibrillation, and use of tissue plasminogen activator. For the subgroup analysis of the learning curve, the logarithm values of the procedure time were compared with treatment experience with adjustment for the occluded vessels using linear regression analysis (ANCOVA).

In this study, $p$ values $<0.05$ are considered statistically significant and are expressed at 2 significance levels. If the $\mathrm{p}$ value is $<0.001$, it is expressed as $\mathrm{p}<0.001$.

All analyses were performed using JMP (version 12.2.0) and SAS software (version 9.3, SAS Institute).
TABLE 1. Baseline characteristics of the SR and ADAPT groups

\begin{tabular}{|c|c|c|c|}
\hline Characteristic & $\begin{array}{l}\text { SR } \\
\text { Group }\end{array}$ & $\begin{array}{l}\text { ADAPT } \\
\text { Group }\end{array}$ & $\begin{array}{c}\mathrm{p} \\
\text { Value }\end{array}$ \\
\hline No. of patients & 45 & 44 & \\
\hline Mean age $\pm S D$, yrs & $73.1 \pm 8.7$ & $77.8 \pm 10.6$ & 0.02 \\
\hline Female & $19(42)$ & $20(45)$ & 0.75 \\
\hline Premorbid mRS score 0-1 & $33(75)$ & $33(75)$ & 0.94 \\
\hline $\begin{array}{l}\text { Mean body mass index } \pm \mathrm{SD} \text {, } \\
\mathrm{kg} / \mathrm{m}^{2}\end{array}$ & $22.0 \pm 0.54$ & $22.4 \pm 0.56$ & 0.63 \\
\hline Atrial fibrillation & $24(53)$ & $25(56)$ & 0.74 \\
\hline Hypertension & $23(51)$ & $22(50)$ & 0.91 \\
\hline Diabetes mellitus & $15(33)$ & $9(20)$ & 0.17 \\
\hline $\begin{array}{l}\text { Median time from stroke on- } \\
\text { set to arrival at the hospital } \\
(I Q R), \text { min }\end{array}$ & $104(10-210)$ & $100(30-201)$ & 0.69 \\
\hline $\begin{array}{l}\text { Mean systolic blood pressure } \\
\quad \pm \mathrm{SD}, \mathrm{mm} \mathrm{Hg}\end{array}$ & $155 \pm 4.1$ & $157 \pm 4.2$ & 0.75 \\
\hline $\begin{array}{l}\text { Mean blood glucose level } \pm \\
\quad \mathrm{SD}, \mathrm{mg} / \mathrm{dl}\end{array}$ & $142 \pm 5.6$ & $129 \pm 5.6$ & 0.12 \\
\hline $\begin{array}{l}\text { Median pretreatment NIHSS } \\
\quad(\mathrm{IQR})\end{array}$ & $19(15-26)$ & $17(12-23)$ & 0.15 \\
\hline $\begin{array}{l}\text { Intravenous tissue plasmino- } \\
\text { gen activator }\end{array}$ & $21(46)$ & $23(52)$ & 0.59 \\
\hline Oral anticoagulant & $9(20)$ & $10(22)$ & 0.75 \\
\hline $\begin{array}{l}\text { Administration of intravenous } \\
\text { tissue plasminogen activa- } \\
\text { tor at outside hospital }\end{array}$ & $6(13)$ & $7(15)$ & 0.73 \\
\hline Wake-up stroke & $5(11)$ & $6(13)$ & 0.71 \\
\hline Occluded vessel & & & 0.23 \\
\hline ICA & $6(13)$ & $11(25)$ & \\
\hline$M_{1}$ & $26(57)$ & $18(40)$ & \\
\hline $\mathrm{M}_{2}$ & $7(15)$ & $9(20)$ & \\
\hline Basilar artery & $4(8)$ & $6(13)$ & \\
\hline Posterior cerebral artery & $2(4)$ & $0(0)$ & \\
\hline Tandem lesion & $2(4)$ & $6(13)$ & 0.12 \\
\hline
\end{tabular}

Values are shown as the number of patients (\%) unless indicated otherwise.

\section{Results}

\section{Baseline Characteristics}

Between November 2013 and January 2016, a total of 96 patients with acute ischemic stroke due to major artery occlusion underwent endovascular treatment at our institution. Among these, 7 patients $(8.3 \%)$ were excluded because of balloon angioplasty (2 patients), carotid artery stenting (2 patients), intraarterial thrombolysis ( 2 patients), or guide catheter aspiration (1 patient); thus, 89 patients $(92.7 \%)$ treated with SR or ADAPT were included in this study.

Forty-five patients (of 89 patients [50.5\%]) were treated with SR as the first-line strategy and included in the SR group, and 44 patients (of 89 patients [49.4\%]) were treated with ADAPT as the first-line strategy and included in the ADAPT group. The baseline characteristics of patients included in the present study are listed in Table 1. Patients 
TABLE 2. Outcomes of the SR and ADAPT groups

\begin{tabular}{lcccccc}
\hline \multicolumn{1}{c}{ Outcome } & $\begin{array}{c}\text { SR } \\
\text { Group }\end{array}$ & $\begin{array}{c}\text { ADAPT } \\
\text { Group }\end{array}$ & $\begin{array}{c}\text { Unadjusted OR } \\
(95 \% \mathrm{Cl})\end{array}$ & $\begin{array}{c}\mathrm{p} \\
\text { Value }\end{array}$ & $\begin{array}{c}\text { Adjusted OR } \\
(95 \% \mathrm{Cl})\end{array}$ & $\begin{array}{c}\mathrm{p} \\
\text { Value }\end{array}$ \\
\hline No. of patients & 45 & 44 & & & & \\
\hline Successful recanalization* & $38(84)$ & $28(65)$ & $2.80(1.01-7.77)$ & 0.04 & $4.29(1.30-16.4)$ & 0.01 \\
\hline Additional therapy & $4(9)$ & $14(31)$ & $0.20(0.06-0.69)$ & $<0.01$ & $4.10(1.12-18.4)$ & 0.03 \\
\hline $\begin{array}{l}\text { Median time from puncture to recanalization } \\
\text { or completion of procedure (IQR), min }\end{array}$ & $42(23-69)$ & $76(44-124)$ & $\mathrm{NA}$ & 0.004 & $\mathrm{NA}$ & 0.04 \\
\hline Subarachnoid hemorrhage & $7(15)$ & $5(11)$ & $1.43(0.41-4.92)$ & 0.33 & $2.24(0.51-11.5)$ & 0.28 \\
\hline SICH & $1(2)$ & $0(0)$ & $\mathrm{NA}$ & 0.32 & $\mathrm{NA}$ & 0.99 \\
\hline Good clinical outcome at 90 days† & $22(49)$ & $15(34)$ & $1.84(0.78-4.34)$ & 0.15 & $1.32(0.48-3.63)$ & 0.58 \\
\hline Median 90-day mRS score (IQR) & $3(1-4)$ & $4(2-5)$ & $\mathrm{NA}$ & 0.15 & $\mathrm{NA}$ & 0.70 \\
\hline
\end{tabular}

NA = not applicable.

Values are shown as the number of patients (\%) unless indicated otherwise.

* Defined as $\mathrm{mTICl}$ score $2 \mathrm{~b}-3$.

$\dagger$ Defined as mRS score $0-2$.

in the SR group were younger than those in the ADAPT group (mean \pm SD $73.1 \pm 8.7$ years vs $77.8 \pm 10.6, \mathrm{p}=$ $0.02)$. The ADAPT group had relatively more ICA occlusions (25\% vs $13 \%$ ), fewer $\mathrm{M}_{1}$ occlusions ( $40 \%$ vs $57 \%$ ), and more tandem occlusions than the SR group (13\% vs $4 \%, \mathrm{p}=0.12)$; however, these differences were not significant.

\section{Procedural and Clinical Results in All Patients}

In the SR group, the first SR used was the Solitaire FR in 42 patients (93\%), and the Trevo ProVue retriever was used in 2 patients (4.4\%). SR could not be deployed in 1 patient $(2.2 \%)$.

In the ADAPT group, the largest aspiration catheter that could be advanced to the thrombus was used. The 5MAX or 5MAX ACE catheter was engaged to the thrombus in 26 patients $(58 \%)$. Additional therapy was required in 14 patients $(31 \%)$. The aspiration catheter could not be advanced in the occluded vessel in 1 patient $(2.2 \%)$.

Among the entire study population, the SR group had a higher recanalization rate (mTICI $2 b-3$ in $84 \%$ vs $65 \%$ of patients, $\mathrm{p}=0.01$ ) and a shorter procedure time (median 42 minutes vs 76 minutes, $p=0.03$ ). Hemorrhagic complica-

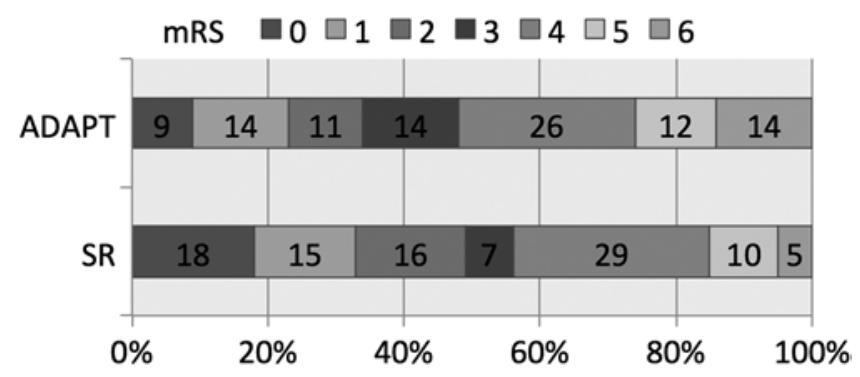

FIG. 1. Functional outcomes at 90 days in patients treated with ADAPT or SR. The percentages of patients with $\mathrm{mRS}$ scores at 90 days are presented. Patients treated with SR achieved relatively better clinical outcomes than those treated with ADAPT, although this was not statistically significant (mRS score $0-2$ at 90 days, $49 \%$ vs 34\%, respectively; $p=$ $0.58)$. The shift analysis was also not statistically significant $(p=0.70)$. tions were rare in both groups (SICH in $2 \%$ vs $0 \%$ of patients, $p=0.28$ ). The percentage of patients who required additional therapy was higher in the ADAPT group than in the SR group (9\% vs 31\%; $p=0.03$ ). The differences in the recanalization rate, procedure time, and additional therapy were persistent after adjustment for age, site of the occluded vessels, presence of tandem occlusion, arterial fibrillation, and use of tissue plasminogen activator (Table 2).

The rate of good clinical outcome at 90 days, although not statistically significant, was relatively better in the SR group than in the ADAPT group (49\% vs $34 \%, \mathrm{p}=0.58$ ). The shift toward better outcomes between the 2 groups was also statistically insignificant $(\mathrm{p}=0.70)$ (Table 2 and Fig. 1).

\section{Learning Curve Effect on Treatment Strategies}

The Endovascular Team Experience

The SR and ADAPT groups were divided into 3 sequential subgroups to assess the learning curve effects on procedural and clinical outcomes in the same endovascular team. There were 15 patients in all subgroups except the third ADAPT subgroup, which consisted of 14 patients. Among the patients treated with SR, successful recanalization was achieved in 14 (93\%), 11 (73\%), and 13 patients $(86 \%)$ in the first, second, and last treatment subgroups, respectively (Fig. 2). The median procedure times were 60, 47, and 21 minutes in the first, second, and third subgroups (Fig. 3). SICH was observed in only 1 patient in the last subgroup.

Among the patients treated with ADAPT, successful recanalization was achieved in $9(60 \%), 10(66 \%)$, and 10 patients (71\%) in the first, second, and third treatment subgroups (Fig. 2). The median procedure times were 93, 69, and 66 minutes in the first, second, and third treatment subgroups, respectively. SICH was not observed in any of the patients treated with ADAPT (Fig. 3).

On the regression analysis, according to the coefficient for the treatment experience, improvement in procedure time was greater in the SR group $(p=0.01)$ even after adjusting for the occluded vessels. 


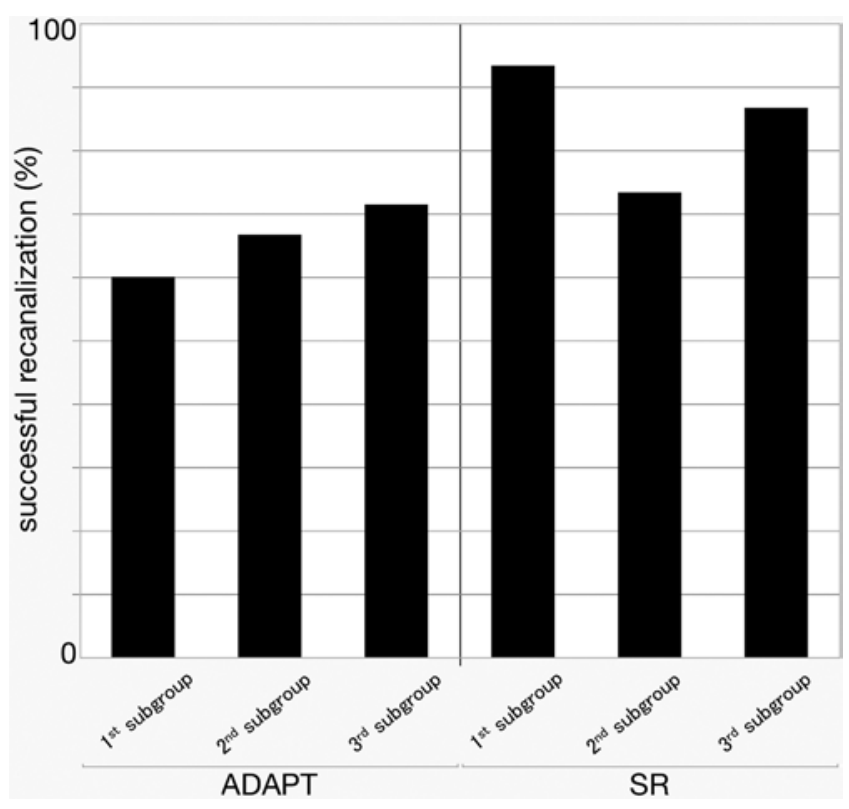

FIG. 2. The shift in the recanalization rate with the team's experience. The rate of successful recanalization ( $\mathrm{mTICl} 2 \mathrm{~b}-3$ ) was represented by 3 sequential groups of patients treated with ADAPT or SR. Recanalization rates gradually increased in the ADAPT subgroups, whereas the recanalization rates in the SR subgroups were already high in the initial group.

\section{Operator Experience}

The endovascular team consisted of 9 operators, all of whom were neurointerventional specialists. The median number of patients treated by each operator was the same and very small in both groups (median [IQR] 3 [2-5] in both groups). Similar to the assessment of the learning curve for the team's experience, the SR and ADAPT groups were divided into 3 sequential subgroups. The first subgroup consisted of patients who were the first and second patients treated by each operator, the second subgroup consisted of patients who were the third and fourth patients treated by each operator, and the third subgroup consisted of patients who were the fifth and subsequent patients treated by each operator. In the SR group, successful recanalization was achieved in 14 of 17 patients (82\%), 12 of 14 patients $(85 \%)$, and 12 of 14 patients $(85 \%)$ in the first, second, and third treatment subgroups, respectively. The median procedure times were 62,42 , and 27 minutes in the first, second, and third subgroups, respectively (Fig. 4).

Among the patients treated with ADAPT, successful recanalization was achieved in 11 of 17 patients (64\%), 9 of 14 patients (64\%), and 9 of 13 patients $(69 \%)$ in the first, second, and third treatment subgroups. The median procedure times were 91, 99, and 53 minutes in the first, second, and third treatment subgroups, respectively (Fig. 4).

On the regression analysis, according to the coefficient for treatment experience, improvement in procedure time was better in the SR group than the ADAPT group $(\mathrm{p}<$ 0.01 ) even after adjustment for the occluded vessels. Hemorrhagic complications were rare in both groups, as described above.

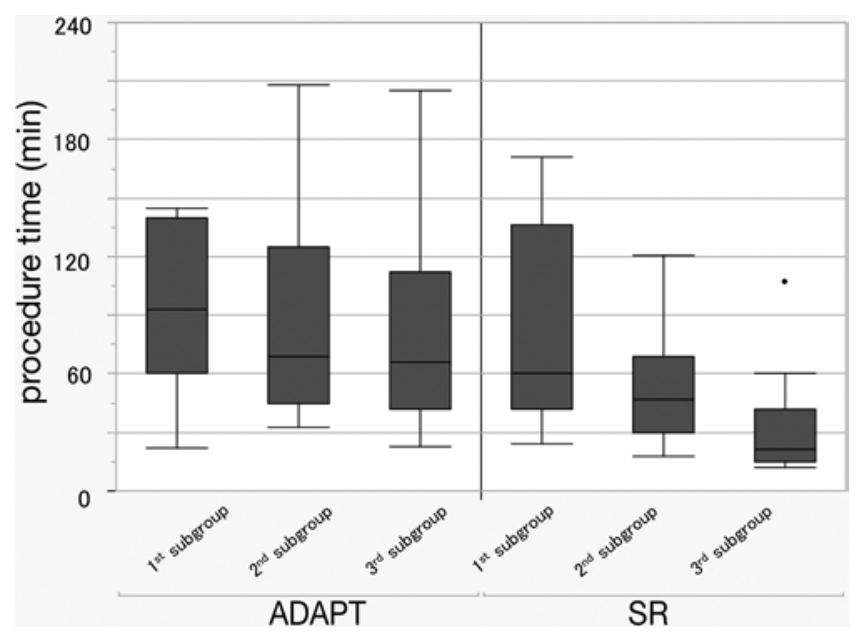

FIG. 3. Comparison of improvements in procedure time with the team's experience. The box-and-whiskers plots show the median (horizontal line), IQR (box), range (whiskers), and outlier (dot) for procedure time (time from puncture to recanalization or completion of the procedure). The procedure time is represented in 3 sequential groups of patients treated with ADAPT or SR. The SR group showed a more rapid improvement in time than the ADAPT group on the regression analysis ( $p$ $=0.01$ ).

\section{Discussion}

In the present study, we found that SR was associated with higher recanalization rates, shorter procedure times, and less additional therapy than ADAPT and that hemorrhagic complications were rare with both treatment approaches. Assessment of the learning curve revealed that higher recanalization rates could be achieved with less ex-

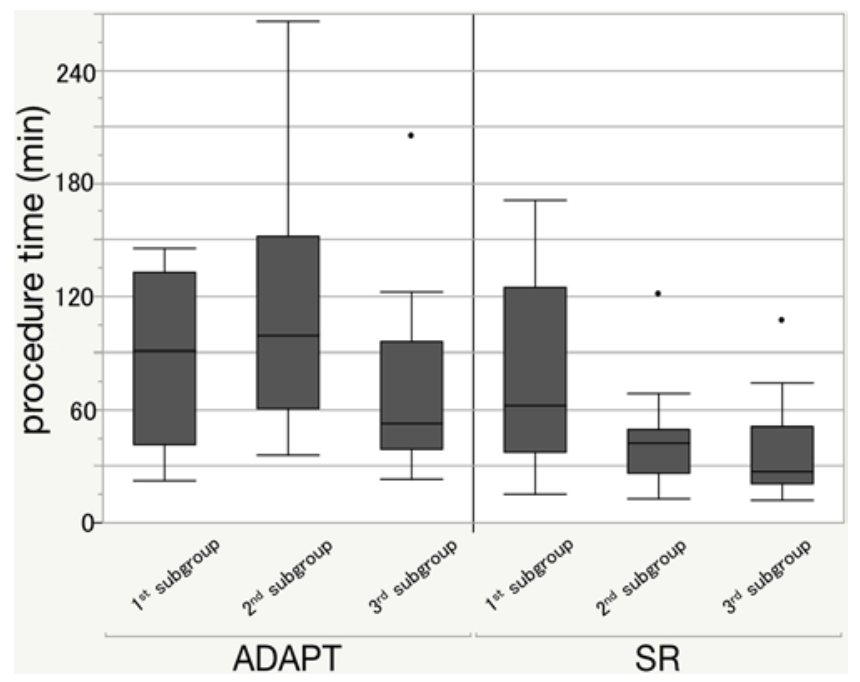

FIG. 4. Comparison of improvements in procedure time with each operator's experience. The box-and-whiskers plots show the median (horizontal line), IQR (box), range (whiskers), and outliers (dots) of the procedure time (time from puncture to recanalization or completion of the procedure). The procedure time is represented in 3 sequential groups of patients treated with ADAPT or SR. The SR group showed a more rapid improvement in time than the ADAPT group on the regression analysis $(p<0.01)$. 
perience and that procedure times dramatically decreased with SR. In contrast, the gradual improvements in the recanalization rates and procedure times with the use of ADAPT over time were relatively limited compared with those achieved with SR.

Although we compared the procedural and clinical outcomes in the initial experiences using the standard SR technique and ADAPT at our institution, the recanalization and complication rates as well as the clinical outcome of the patients treated with SR were within the range of the results reported in previous multicenter randomized clinical trials. ${ }^{2,5,8,9,14}$ Conversely, in the current study, the recanalization rate and clinical outcome of the patients treated with ADAPT were inferior to those reported in previous studies. $1,10,16,17-19$

A few retrospective studies have compared the procedural and clinical outcomes of SR and ADAPT. Turk et al. found that ADAPT and SR with local aspiration had similar recanalization rates (78\% vs $80 \%)$ and good clinical outcomes at 90 days (47\% vs 43\%). ${ }^{19}$ Almandoz et al. found that the recanalization rates (defined as TICI $2 \mathrm{~b}-3$ and achieved in $84 \%$ and $89 \%$ of patients, respectively) and procedure times (mean time from puncture to reperfusion was 51 minutes and 50 minutes, respectively) were similar between the Solumbra technique and ADAPT; however, complication rates (SICH in $12 \%$ vs $2 \%$ of patients) and clinical outcome (mRS 0-2 at 90 days in $31 \%$ vs $56 \%$ of patients, respectively) were better with ADAPT. ${ }^{1}$ SR with local aspiration and the Solumbra technique share several characteristics. Both use large-bore intermittent catheters (usually Penumbra catheters), and the intermittent catheter-not the guiding catheter-is used for aspiration and stent retrieval in both approaches. However, techniques that use intermittent catheters are not a routine part of the standard SR technique. Recently, Lapergue et al. compared the standard SR technique with Solitaire FR and ADAPT. ${ }^{10}$ They reported that ADAPT was associated with a higher recanalization rate than SR (mTICI $2 b-3$ achieved in $82.3 \%$ vs $68.9 \%$ of patients, respectively; $p=0.02$ ) and that the procedure times and clinical outcomes were similar (median procedure times of 45 minutes vs 50 minutes, $\mathrm{p}=0.42$; mRS $0-2$ at 90 days achieved in $53 \%$ vs $54 \%$, respectively, $p=0.79$ ). This was the first study to compare efficacy and safety between the standard SR technique and ADAPT. Importantly, these findings were obtained from 2 high-volume stroke centers and consisted of data for more than 200 treated cases, which is a large number for a relatively small stroke center like our institution.

There are several reports on the learning curves of neuroendovascular treatment approaches such as aneurysm coil embolization, ${ }^{3,15}$ intracranial stenting, ${ }^{11,22}$ carotid artery stenting, $7,12,20$ and mechanical thrombectomy. 4,6 Among these reports, some focused on the specific operator's experience $e^{15,20}$ and others focused on the team's or institutional experience. ${ }^{3,4,6,7,11,12,22}$ According to these reports, both the individual operator's experience and team's experience are associated with improvement in procedural and clinical outcomes.

Benardete et al. reported improvements in functional outcomes in mechanical thrombectomy from the 1st year to 2 nd year at a newly organized, comprehensive stroke center. ${ }^{4}$ While they reported that the percentage of patients with mRS scores of 0-2 at discharge improved from 9\% to $57 \%$ in a total of 18 patients treated over 2 years, technical details of the endovascular strategies used were not disclosed. Eesa et al. analyzed the learning curve for the Solitaire stent ${ }^{6}$ that was used in 83 patients who were divided into 3 groups and reported that the mean time from puncture to recanalization decreased by 43 minutes from 84 minutes with experience in the first subgroup to 41 minutes in the last subgroup. Our results in patients treated with SR were similar: there was a 39-minute decrease in procedure time during the course of treatment among approximately 40 patients. While Eesa et al. did not report the effect of the learning curve on recanalization rates, we found that high recanalization rates (mTICI $2 b-3$ achieved in $>80 \%$ of patients) could be achieved with less experience using SR compared with ADAPT. These results should encourage relatively small stroke centers to implement mechanical thrombectomy with SR.

There are no reports that investigate the effects of the learning curve on ADAPT outcomes. We found that the initial outcome with ADAPT was inferior to those observed by high-volume centers.,10,16,17-19 Despite a trend toward gradual improvement, the results in the present study are very different from the findings of high-volume centers. The results imply that ADAPT required more experience (i.e., improvement in technical aspects) than SR to achieve good clinical outcomes, which is comparable to results observed at high-volume centers.

This study suggests that there are learning curve effects for the team and operator in mechanical thrombectomy. Although the study period exceeded 2 years, the number of cases treated by each operator was small (5 cases on average). Given that learning curve effects exist for each operator's experience, limiting the number of operators might have improved our clinical outcomes. However, our results suggest that, although the individual operators' experiences were limited, the team's experience (including the approach to teamwork and sharing of technical tips) might have compensated for individual experiences and improved the procedural outcomes, especially when using SRs.

This is the first report to compare the learning curves of SR and ADAPT. Our results show that SR had more rapid improvement in procedure time from the initial experience, even after adjustment for the site of the occluded vessels. Despite the potential benefits and better clinical outcomes observed with ADAPT than SR at high-volume centers, ADAPT requires more experience. If ADAPT is to be considered the first-line endovascular strategy of choice for acute ischemic stroke, some comprehensive stroke centers likely need a number of years to improve ADAPT outcomes and reach the level achieved at highvolume centers.

\section{Study Limitations}

There are several limitations in the present study. First, this was a retrospective study at a single institution, and the data were collected in an unblinded manner. Although we assessed each device as a first-line treatment approach in a specific time period, potential bias in pa- 
tient characteristics should be acknowledged. Specifically, the proportion of patients with ICA occlusion was higher (25\% vs $13 \%$ ) and the proportion with $\mathrm{M}_{1}$ occlusion was lower (40\% vs 57\%) in the ADAPT group than in the SR group, which might have influenced the procedural and clinical outcomes. However, the difference in the procedural outcomes was persistent after adjusting for several confounding factors, i.e., age, site of the occluded vessels, presence of tandem occlusion, arterial fibrillation, and use of tissue plasminogen activator. In addition, the procedural outcomes were better for both ICA and $\mathrm{M}_{1}$ cases in the SR group. Second, since a relatively small number of patients were enrolled in the present study, there was a possibility of a type II error in the statistical analysis. Finally, we compared our initial experiences with SR and ADAPT, and procedure outcomes were still improving in both groups. A currently ongoing randomized clinical trial comparing SR and ADAPT will confirm the efficacy of these 2 treatment strategies.

\section{Conclusions}

In our initial experience, SR had a higher recanalization rate and shorter procedure time than ADAPT in treating acute ischemic stroke due to large vessel occlusion. These differences were persistent after adjusting for covariate factors, especially the location of the occluded vessel. Additionally, a high recanalization rate was achieved with less experience in SR, and the procedure time dramatically decreased with experience. The recanalization rate and procedure time gradually improved with ADAPT, albeit to a more limited extent than SR. These observed effects of the learning curve on both techniques might be useful when choosing the first-line approach for initial endovascular treatment of acute ischemic stroke at relatively small stroke centers.

\section{References}

1. Almandoz JED, Kayan Y, Young ML, Fease JL, Scholz JM, Milner AM, et al: Comparison of clinical outcomes in patients with acute ischemic strokes treated with mechanical thrombectomy using either Solumbra or ADAPT techniques. J Neurointerv Surg 8:1123-1128, 2016

2. Berkhemer OA, Fransen PS, Beumer D, van den Berg LA, Lingsma HF, Yoo AJ, et al: A randomized trial of intraarterial treatment for acute ischemic stroke. N Engl J Med 372:11-20, 2015

3. Brinjikji W, Rabinstein AA, Lanzino G, Kallmes DF, Cloft HJ: Patient outcomes are better for unruptured cerebral aneurysms treated at centers that preferentially treat with endovascular coiling: a study of the national inpatient sample 2001-2007. AJNR Am J Neuroradiol 32:1065-1070, 2011

4. Benardete EA, Nair AK: Endovascular stroke therapy results improve over time: the 'learning curve' at a new comprehensive stroke center. Cerebrovasc Dis Extra 4:235-242, 2015

5. Campbell BC, Mitchell PJ, Kleinig TJ, Dewey HM, Churilov L, Yassi N, et al: Endovascular therapy for ischemic stroke with perfusion-imaging selection. N Engl J Med 372:10091018, 2015

6. Eesa M, Burns PA, Almekhlafi MA, Menon BK, Wong JH, Mitha A, et al: Mechanical thrombectomy with the Solitaire stent: is there a learning curve in achieving rapid recanalization times? J Neurointerv Surg 6:649-651, 2014

7. Fiehler J, Söderman M, Turjman F, White PM, Bakke SJ,
Mangiafico S, et al: Future trials of endovascular mechanical recanlisation therapy in acute ischemic stroke patients - a position paper endorsed by ESMINT and ESNR. Part II: methodology of future trials. Neuroradiology 54:1303-1312, 2012

8. Goyal M, Demchuk AM, Menon BK, Eesa M, Rempel JL, Thornton J, et al: Randomized assessment of rapid endovascular treatment of ischemic stroke. N Engl J Med 372:10191030,2015

9. Jovin TG, Chamorro A, Cobo E, de Miquel MA, Molina CA, Rovira A, et al: Thrombectomy within 8 hours after symptom onset in ischemic stroke. N Engl J Med 372:2296-2306, 2015

10. Lapergue B, Blanc R, Guedin P, Decroix JP, Labreuche J, Preda C, et al: A direct aspiration, first pass technique (ADAPT) versus stent retrievers for acute stroke therapy: an observational comparative study. AJNR Am J Neuroradiol 37:1860-1865, 2016

11. Nahab F, Lynn MJ, Kasner SE, Alexander MJ, Klucznik R, Zaidat OO, et al: Risk factors associated with major cerebrovascular complications after intracranial stenting. Neurology 72:2014-2019, 2009

12. Parlani G, De Rango P, Verzini F, Cieri E, Simonte G, Casalino A, et al: Safety of carotid stenting (CAS) is based on institutional training more than individual experience in largevolume centres. Eur J Vasc Endovasc Surg 45:424-430, 2013

13. Powers WJ, Derdeyn CP, Biller J, Coffey CS, Hoh BL, Jauch EC, et al: 2015 American Heart Association/American Stroke Association focused update of the 2013 guidelines for the early management of patients with acute ischemic stroke regarding endovascular treatment: a guideline for healthcare professionals from the American Heart Association/American Stroke Association. Stroke 46:3020-3035, 2015

14. Romano DG, Cioni S, Vinci SL, Pero G, Comelli C, Comai A, et al: Thromboaspiration technique as first approach for endovascular treatment of acute ischemic stroke: initial experience at nine Italian stroke centers. J Neurointerv Surg 9:6-10, 2017

15. Saver JL, Goyal M, Bonafe A, Diener HC, Levy EI, Pereira VM, et al: Stent-retriever thrombectomy after intravenous t-PA vs. t-PA alone in stroke. N Engl J Med 372:2285-2295, 2015

16. Singh V, Gress DR, Higashida RT, Dowd CF, Halbach VV, Johnston SC: The learning curve for coil embolization of unruptured intracranial aneurysms. AJNR Am J Neuroradiol 23:768-771, 2002

17. Turk AS, Spiotta A, Frei D, Mocco J, Baxter B, Fiorella D, et al: Initial clinical experience with the ADAPT technique: a direct aspiration first pass technique for stroke thrombectomy. J Neurointerv Surg 6:231-237, 2014

18. Turk AS, Frei D, Fiorella D, Mocco J, Baxter B, Siddiqui A, et al: ADAPT FAST study: a direct aspiration first pass technique for acute stroke thrombectomy. J Neurointerv Surg 6:260-264, 2014

19. Turk AS, Turner R, Spiotta A, Vargas J, Holmstedt C, Ozark $\mathrm{S}$, et al: Comparison of endovascular treatment approaches for acute ischemic stroke: cost effectiveness, technical success, and clinical outcomes. J Neurointerv Surg 7:666-670, 2015

20. Verzini F, Cao P, De Rango P, Parlani G, Maselli A, Romano $\mathrm{L}$, et al: Appropriateness of learning curve for carotid artery stenting: an analysis of periprocedural complications. J Vasc Surg 44:1205-1212, 2006

21. Wahlgren N, Ahmed N, Dávalos A, Ford GA, Grond M, Hacke W, et al: Thrombolysis with alteplase for acute ischaemic stroke in the Safe Implementation of Thrombolysis in Stroke-Monitoring Study (SITS-MOST): an observational study. Lancet 369:275-282, 2007 
22. Yu SCH, Leung TWH, Lee KT, Wong LK: Learning curve of Wingspan stenting for intracranial atherosclerosis: singlecenter experience of 95 consecutive patients. J Neurointerv Surg 6:212-218, 2014

23. Zaidat OO, Yoo Aj, Khatri P, Tomsick TA, von Kummer R, Saver JL, et al: Recommendations on angiographic revascularization grading standards for acute ischemic stroke: a consensus statement. Stroke 44:2650-2663, 2013

\section{Disclosures}

The authors report no conflict of interest concerning the materials or methods used in this study or the findings specified in this paper.

\section{Author Contributions}

Conception and design: Nishi, Ishii. Acquisition of data: Nishi, Ishii, Nakahara, Matsumoto, Sadamasa, Kai, Ishibashi, Nagata. Analysis and interpretation of data: Nishi, Yamamoto, Morita. Drafting the article: Nishi. Critically revising the article: Nishi. Reviewed submitted version of manuscript: Nishi, Ishii, Nakahara, Matsumoto, Sadamasa, Kai, Ishibashi, Yamamoto, Nagata. Statistical analysis: Yamamoto, Morita. Study supervision: Ishii, Nagata.

\section{Correspondence}

Hidehisa Nishi: Kyoto University Hospital, Sakyo-ku, Kyoto, Japan.venturahighway83@gmail.co. 